\title{
Development of a Novel Tracking System for Photovoltaic Efficiency in Low Level Radiation
}

\author{
Jung-Sik Choi*, Jae-Sub Ko*, and Dong-Hwa Chung ${ }^{\dagger}$ \\ ${ }^{\dagger *}$ Dept. of Electrical Control Eng., Sunchon National University, Korea
}

\begin{abstract}
This paper proposes a novel tracking algorithm considering radiation to improve the power of a photovoltaic (PV) tracking system. The sensor method used in a conventional PV plant is unable to track the sun's exact position when the intensity of solar radiation is low. It also has the problem of malfunctions in the tracking system due to rapid changes in the climate. The program method generates power loss due to unnecessary operation of the tracking system because it is not adapted to various weather conditions. This tracking system does not increase the power above that of a power of tracking system fixed at a specific position due to these problems. To reduce the power loss, this paper proposes a novel control algorithm for a tracking system and proves the validity of the proposed control algorithm through a comparison with the conventional PV tracking method.
\end{abstract}

Key Words: Efficiency, Photovoltaic, Power loss, Tracking system, Sensor method, Sun position

\section{INTRODUCTION}

Recently, PV systems have received a great deal of attention due to the exhaustion of energy sources, high oil prices and environmental pollution. A PV system does not require fuel because it directly converts solar energy into electric energy. Also, it is provides a viable means of power generation for applications like power supplies to residential appliances, telecommunications, refrigeration and water pumping. A PV system does not need a power transmission system and its operation and maintenance costs are very low. These systems operate silently and create no atmospheric pollution. Their major defect is that they have a high capital cost. However, with the continuous decrease in the cost of solar cells, there is an increasing interest in PV system applications. A PV system requires special design considerations due to the varying nature of the solar power resulting from unpredictable and sudden changes in weather conditions which change the radiation level as well as the cell operating temperature. Owing to changes in the solar radiation energy and the cell operating temperature, the output power of a solar array is not always constant [1],[2]. Hence, to increase economic feasibility above that of tradition generation systems, a great deal of research on increasing solar cell efficiency, reducing the cost of module production, power converting techniques, solar tracking devices and other related techniques is needed. A fuzzy based program method was proven to be excellence in generation efficiency through realized system and performances analysis [3],[4]. Also, tracking systems using photo sensors and pyranometers were proposed

Manuscript received Dec. 7, 2009; revised May 7, 2010

$\dagger$ Corresponding Author: hwa777@scnu.ac.kr

Tel: +82-61-750-3543, Fax: +82-61-752-1103, Sunchon Nat'l Univ

* Dept. of Electrical Control Eng., Sunchon National Univ., Korea
[5],[6]. A tracking system was applied to a hybrid system which tracks by a calculation of the azimuth and altitude and a photo sensor for a parabolic dish tracking system using a solar collector system that requires a high temperature about $650^{\circ} \mathrm{C} \sim 1200^{\circ} \mathrm{C}$ [7],[8]. Photo sensor tracking systems have difficulty in accurately tracking the sun because the tracking devices experience malfunctions due to rapidly changing weather conditions such as clouds and fog. Furthermore, they move by compulsion to a setting position or stop at the present position when low radiation is encountered. A program method using an astronomy algorithm generates a power loss due to unnecessary operation of the tracking device system because it is unable to cope with the weather conditions. There is a great deal of research on improving the accuracy of tracking solar position. However, research on the unnecessary power loss of tracking devices due to the climate environment has not yet been studied.

Therefore, this paper proposes a novel tracking method that takes into consideration changes in weather conditions using a radiation sensor, a wind speed sensor and a rainfall cumulating sensor. The validity of this tracing method has been proved through experiment results.

\section{Construction of A PV Tracking System}

Divided by installation types, PV arrays can consists of fixed or tracking PVs. Fig. 1 presents a fixed PV system and Fig. 2 shows a slope variable type PV system. The fixed type is the most general format of PV array in a PV plant. This type is installed on a fixed structure and the installation variables are the tilt angle and the azimuth regarding the latitude of the PV plant location. The installation variables of the slope 


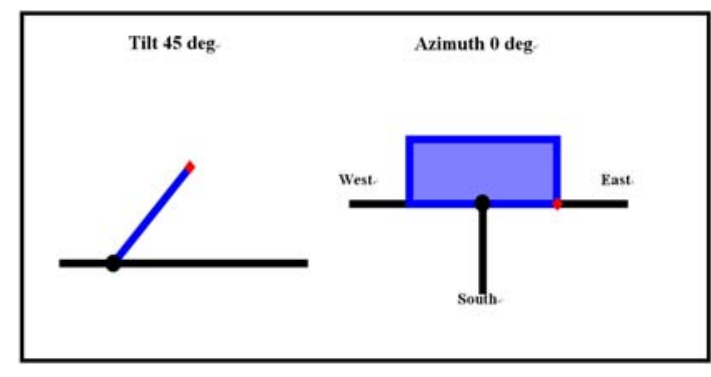

Fig. 1. Fixed generation system of PV system.

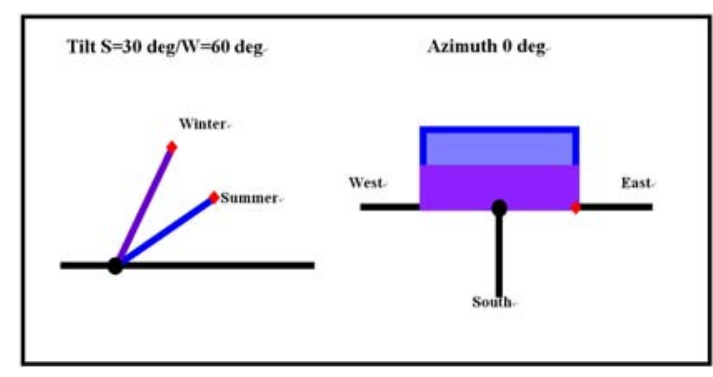

Fig. 2. Slope variable type generation system of PV system.

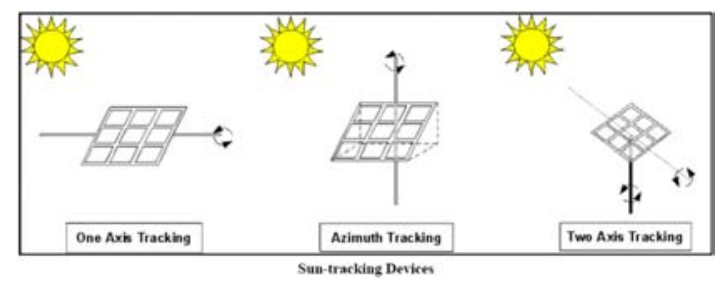

Fig. 3. Type of tracking equipment of PV system.

variable type are the azimuth and the monthly or season tilt angle regarding the latitude of the PV plant location.

A tracking device for a PV system is a structure supporting the PV array. As the incidence angle of the solar radiation energy at the array surface is minimized, the radiation energy which is absorbed becomes greatest. As a result, the power generation efficiency of the PV system is increased [7]-[11]. Types of tracking devices are one axis tracking, azimuth tracking and two axis tracking.

The one axis tracking is tracked by rotation of an axis located at the back side of the array. Installation variables of the axis are the maximum and minimum tilt angle and the azimuth or altitude of the installed rotation axis. This tracking requires a great deal of investigation because the power generation efficiency is reduced by the shadow effect and the area of the PV plant according to the tilt angle and the installation azimuth. Azimuth tracking is a fixed tilt angle considering the latitude of the PV plant installation location. The installation variables of azimuth tracking are the installation tilt angle, the azimuth and the rotation angle while the rotation axis rotates about the vertical axis. Two axis tracking has two rotation axes which are controlled in order to absorb the sun rays when they are perpendicular to the array. Fig. 3 presents one axis tracking, azimuth tracking and two axis tracking, while Table 1 shows their installation variables.
TABLE I

INSTALLATION VARIATION

\begin{tabular}{|c|c|}
\hline Tracking Mode & Parameters required \\
\hline \hline No tracking & Tilt and azimuth of PV array \\
\hline $\begin{array}{c}\text { Seasonal tilt } \\
\text { adjustment }\end{array}$ & Tilt and azimuth of PV array \\
\hline 1-axis tracking & Tilt or azimuth of tracking axes \\
\hline 1-axis tracking & None \\
\hline Azimuth tracking & Tilt and azimuth of tracking axes \\
\hline
\end{tabular}

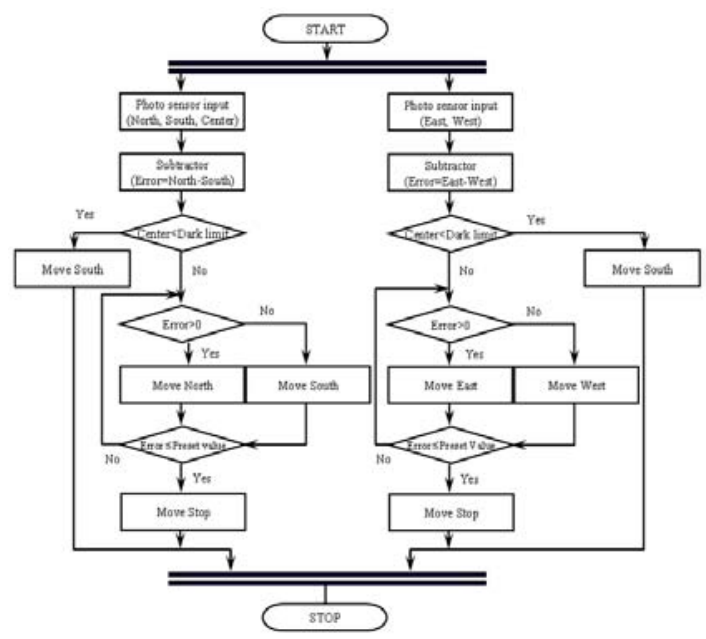

Fig. 4. Control algorithm of photo sensor tracking method.

\section{Control Algorithm of the Tracking System}

Control methods for the tracking controller are the program tracking system, the photo sensor tracking system and the hybrid method which is a mixed program and photo sensor tracking systems.

\section{A. Photo Sensor Tracking Method}

The photo sensor tracking method is operated by sensor error. In conditions of low radiation, the tracking device is forced to moved to a set position or malfunction.

\section{B. Program Tracking Method}

The program tracking method calculates the solar position to control the position of the PV array by a real-time calculation or a predefined table using a motion equation of the Earth as defined in astronomy. Devices utilizing the program method consist of sensors in which we can confirm the location of the tracing device, a controller within a microprocessor, a motor and a decelerator. The program method is controlled independently from weather changes and can be controlled to a desired positions after sunset. However, this method needs accurate azimuth settings and compensation. Furthermore, this method has a problem that deceases the power generation efficiency by operating the tracking devices regardless of the weather and when the intensity of radiation is low.

1) Calculation of Solar Position: The energy generated from solar cells is proportional to the amount of incident light. It reaches its maximum value when the solar cell is perpendicular to the sun's rays. The Earth revolves in an counterclockwise direction $1^{\circ}$ per day around the sun and it 


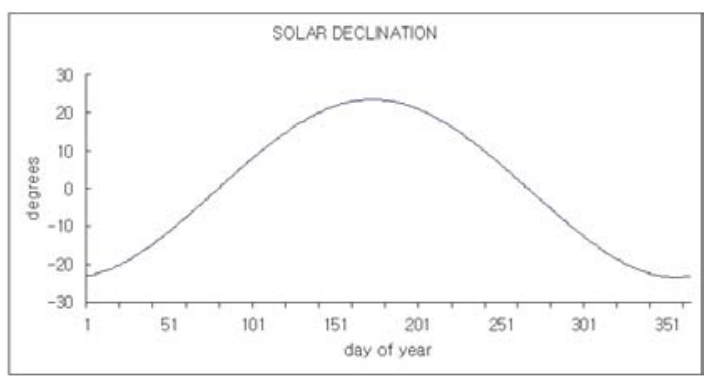

Fig. 5. Declination variation of solar during 1 year.

rotates from west to east around a revolving axis in which it declines $23.45^{\circ}$. Because of this, the latitude and longitude of the location as well as the date and time are needed to calculate the azimuth and the altitude of the sun. Accordingly, the declination, equation of time, true solar time and hour angle of the sun have to be calculated.

Declination is the angle formed between a straight line from the central axis of the earth to the central axis of the sun and the plane of the equator and it is expressed as follows:

$$
\begin{aligned}
& \delta=0.322003-22.9711 \cos (t)-0.14398 \cos (3 t) \\
& +3.946380 \sin (t)+0.019334 \sin (2 t) \\
& +0.059280 \sin (3 t)
\end{aligned}
$$

where, $t$ is day angle. The declination changes daily because the revolving axis of the Earth inclines with 23.45. It varies within a range of $\pm 23.45^{\circ}$ at both ends and has a positive value at the north. Fig. 5 shows the declination change curve at $35^{\circ}$ north latitude and $126.7^{\circ}$ east longitude over a year.

The solar hour angle is the angle between hour circles after meridian where the solar cell module is located. The angle measured at the south point to the westward direction is called the hour angle $\omega$ and is expressed as follows:

$$
\begin{aligned}
& \omega=\left(T_{A S}-12\right) \times 15^{\circ} \\
& =\left(T_{S T D}+\frac{L_{S T}-L_{L O C}}{15}+E O T-12\right) \times 15^{\circ} \\
& T_{A S}: \text { true solar time, } T_{S T D}: \text { local standard time, } \\
& L_{L O C}: \text { inclination of standard meridian, } \\
& L_{L O C}: \text { inclination of the earth, EOT }: \text { equation of time }
\end{aligned}
$$

The equation of time is the time difference between the true solar time and the mean solar time and it is expressed as follows:

$$
\begin{aligned}
& E O T=0.258^{*} \cos (t)-7.416^{*} \sin (t) \\
& -3.648^{*} \cos (2 t)-9.228^{*} \sin (2 t)
\end{aligned}
$$

With the declination and the hour angle from (1) (3), the altitude and the azimuth is expressed as follows:

$$
\begin{aligned}
& \alpha_{S}=\sin ^{-1}(\sin L \sin \delta+\cos L \cos \delta \cos \omega) \\
& \gamma_{S}=\sin ^{-1}\left(\frac{\cos \delta \sin \omega}{\cos \alpha_{S}}\right)
\end{aligned}
$$

$L$ : latitude of the earth
2) PSA Algorithm: The characteristics of the position solar array (PSA) algorithm [12] are as follows:

1) Used together with calendar dates and space time, calculating the Julian date becomes easy.

2) Memory usage is improved with a range of variables and the life span is controlled.

3) Speed and strength are enhanced by a simple and powerful expression due to not needing to calculate the solar azimuth based on the southern and northern hemispheres of the sun.

4) Introduction of coefficients and conditions along with a correction of the time difference helps modify the simple functions of the Nautical Almanac by Michalsky for better accuracy.

The input of the PSA is the time position. The short time among the considering items is the provided date (year, month and day) and the general time (hour, minute and second). The position is given as the angle values of the latitude and longitude at the observing location. Latitude has a positive value in the northern hemisphere and longitude starts from the east. The Julian date is $j d$ and is expressed as follows:

$$
\begin{aligned}
& j d=(1461 \times(y+4800+(m-14) / 12)) / 4 \\
& +(367 \times(m-2-12 \times((m-14 / 12))) / 12 \\
& -(3 \times((y+4900+(m-14) / 12) / 100)) / 4 \\
& +d-32075-0.5+\text { hour } / 24.0
\end{aligned}
$$

where $m$ is the month, $y$ is the year, $d$ is the day of the month, hour is time of the day in normal decimal time. In other words, the function of time contains minutes and seconds, and the numbers below integers are ignored.

The solar ecliptic coordinates can be calculated from the Julian date using (7) (12) (For all radian angles).

$$
\begin{aligned}
& n=j d-2451545.0 \\
& \Omega=2.1429-0.0010394594 \times n \\
& L(\text { mean longitude }) \\
& =4.8950630+0.017202791698 \times n \\
& g(\text { mean anomaly }) \\
& =6.2400600+0.0172019699 \times n \\
& \begin{aligned}
l \text { ecliptic longitude }) & =L+0.03341607 \times \sin (g) \\
& +0.00034894 \times \sin (2 g) \\
& -0.0001134-0.0000203 \\
& \times \sin (\Omega)
\end{aligned} \\
& \text { ep }(\text { obliquity of the ecliptic }) \\
& =0.4090928-6.2140 \times 10^{-9} \times n \\
& +0.0000396 \times \cos (\Omega)
\end{aligned}
$$

Changing from the ecliptic to the sky coordinates is calculated with standard trigonometry and is expressed as follows: 


$$
\begin{aligned}
& r d(\text { right ascension }) \\
& =\tan ^{-1}\left[\frac{\cos (e p) \times \sin (l)}{\cos (l)}\right] \\
& \delta(\text { declination } \mathrm{n})=\sin ^{-1}[\sin (e p) \times \sin (l)] .
\end{aligned}
$$

Changing from the astronomy to the horizontal coordinates is expressed as follows:

$$
\begin{aligned}
& \text { gmst }=6.6974243242 \\
& +0.0657098283 \times n+\text { hour } \\
& \text { lmst }=(\text { gmst } \times 15+\text { Long }) \times(\pi / 180) \\
& \omega(\text { hour angle })=\text { lmst }- \text { ra } \\
& \theta_{z}=\cos ^{-1}[\cos (\Phi) \cos (\omega) \cos (\delta)+\sin (\delta) \sin (\Phi)] \\
& \gamma=\tan ^{-1}\left[\frac{-\sin (\omega)}{\tan (\delta) \cos (\Phi)-\sin (\Phi) \cos (\omega)]}\right. \\
& \text { Parallax }=\frac{\text { EarthMeanRadius }}{\text { AstronomicalUnit }} \times \sin \left(\theta_{z}\right) \\
& \theta_{z}=\theta_{z}+\text { Parallax. }
\end{aligned}
$$

The radius of the Earth and distance to the sun is expressed as follows:

$$
\begin{aligned}
& \text { EarthMeanRadius }=6371.01 \mathrm{~km} \\
& \text { AstronomicalUnit }=149597890 \mathrm{~km} .
\end{aligned}
$$

\section{Control Algorithm with Radiation Variation}

When solar radiation is low because of mist, clouds or rain, the refraction effect of sun's rays affect the PV. Furthermore, the power of the tracking PV system cannot be exceeded when fixed in a particular position despite the tracking system's estimate of azimuth and altitude in real-time. This is because the solar radiation is low and it brings about a power loss in the operating tracking system.

If a sensor tracking system is controlled with little dead band width of the photo sensor, it causes unnecessary power loss by operation of the tracking devices and the generated energy does not increase. This system can reduce the power loss because tracking devices do not operate in the case of a large dead band. However, errors in the azimuth and altitude become far larger, so the power generation efficiency of the PV is decreased.

Therefore, in this paper, while the sun is at a position that can be tracked, an analog value $(05 \mathrm{Vdc})$ of the radiation is input to the $\mathrm{A} / \mathrm{D}$ converter. When it reaches or exceeds a preset value, the proposed method controls the tracking device so that the solar position and the array become perpendicular. If the amount of solar radiation is less than the preset value, the tracking device will be moved to a set position and stop.

The new control method, which takes into consideration radiation change, uses the current azimuth angle, altitude and radiation variables. If the radiation is lower than the value set according to the azimuth and the altitude is within the range that the tracking device can control, the device is forced to reposition and then stop. Furthermore, since the radiation

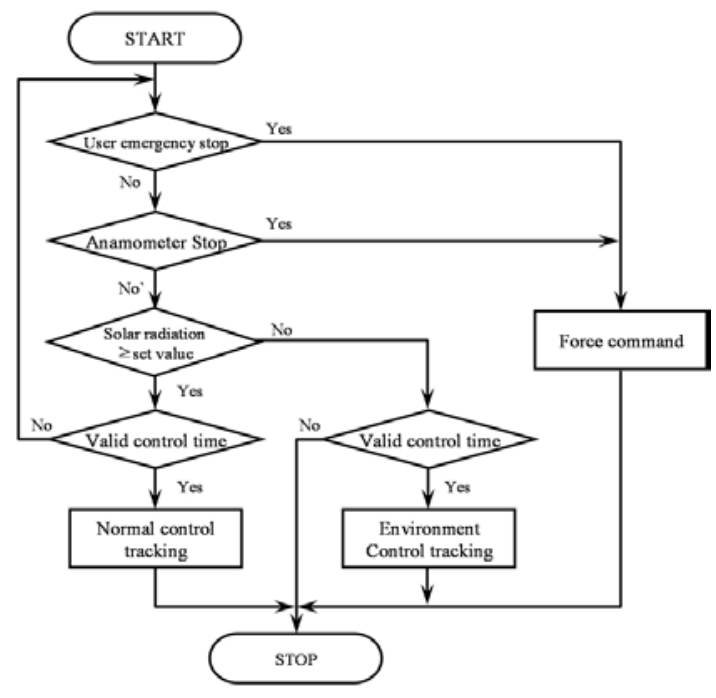

Fig. 6. Control flow-chart by radiation change.

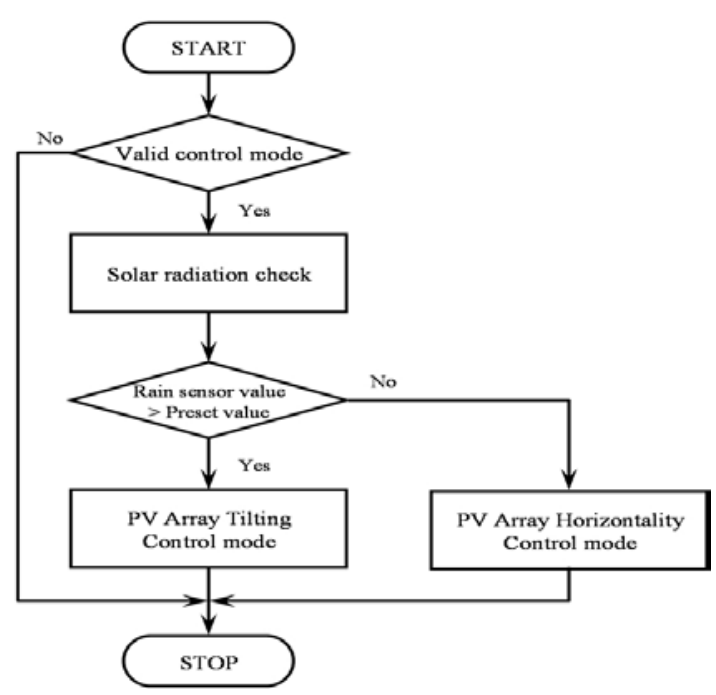

Fig. 7. Control flow-chart by rain sensor change.

has the possibility of making the tracking device malfunction because of clouds, refraction or falling leaves, a certain level of dead band is configured to sample the radiation after a set delay. If the amount exceeds the set value, the tracking device controls the azimuth and the altitude of the sun with the program method.

Fig. 6 and 7 show the control flow chart according to a change in radiation and in the analog input of the rain sensor. If a lower radiation than the set value is input to the controller, the tracking device is switched to environmental control tracking mode. In case of rainfall or snowfall, if the value of the input to the rain sensor is larger than the preset value it repositions the PV array to a preset inclination angle. If rainfall and snowfall cannot be detected, the tracking device moves to a preset position according to the azimuth and altitude. When the radiation exceeds the set value, it is switched to a mode that can control the current azimuth and altitude. 


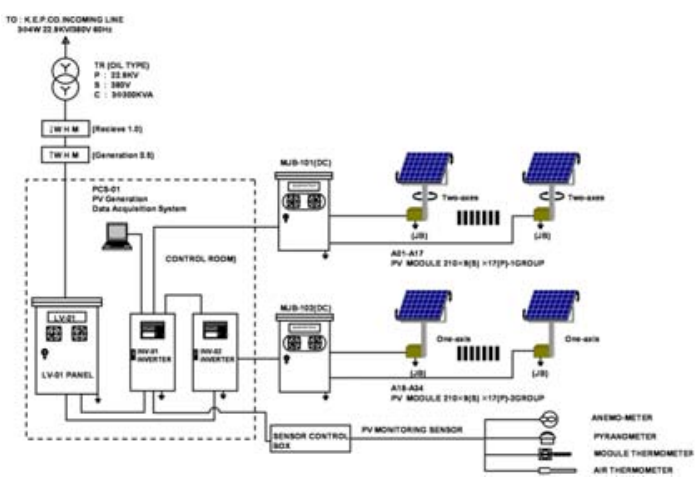

Fig. 8. Configuration of PV generation system.

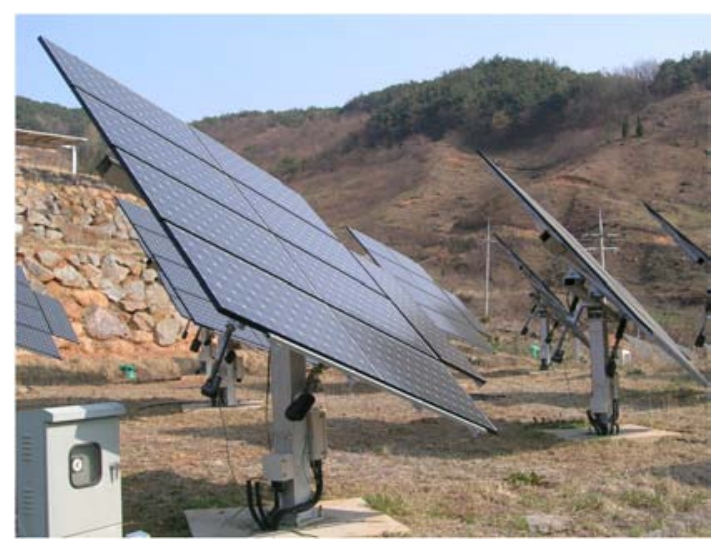

(a) PV tracking system.

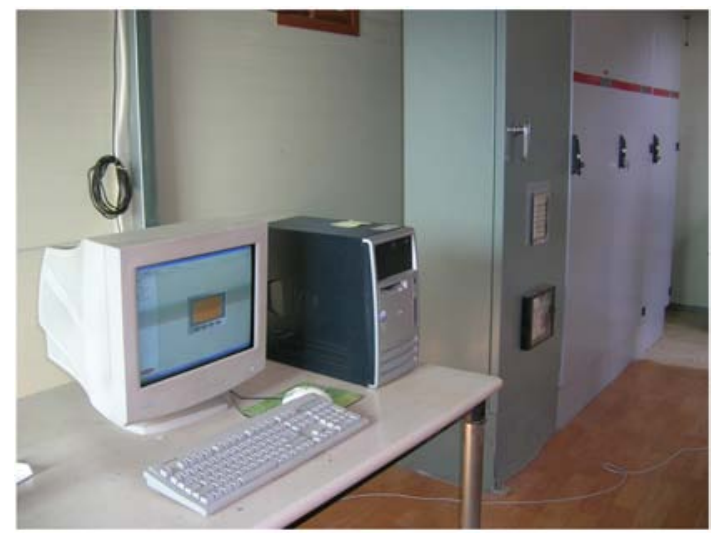

(b) Inverter groups of PV generation.

Fig. 9. Experiment equipment of PV tracking system.

\section{EXPERIMENTAL AND RESUltS}

Fig. 8 presents the configuration of the PV generation system proposed in this paper. Two of the tracking devices are from the 2-axis tracking group and one is from the 1-axis tracking group. The module capacity per group is $32 \mathrm{~kW}$. Fig. 9 is the experiment equipment of the PV generation units. Fig. 9(a) shows the PV tracking system and Fig. 9(b) shows the inverter group of the PV generation system. For a validity analysis of the proposed algorithm, compare the efficiency of the sensor method and the program method during a rapid change in radiation.

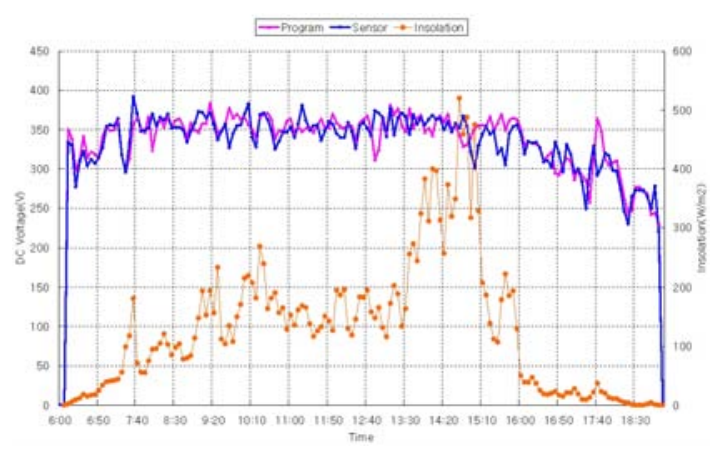

Fig. 10. Change of DC voltage by climate environment (with low radiation).

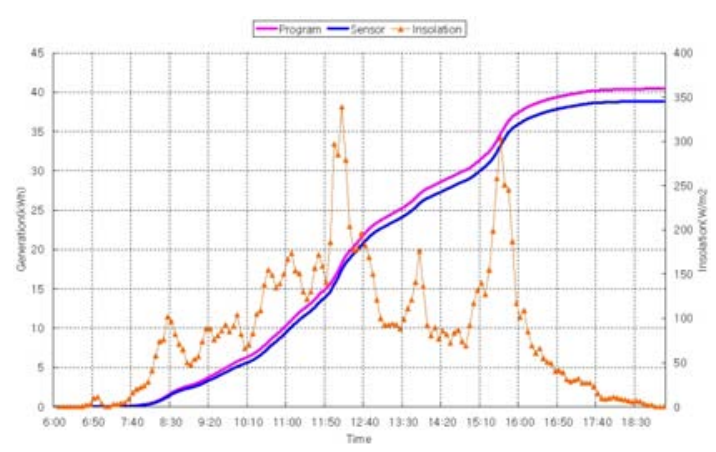

Fig. 11. Comparison with power by control method (with low radiation).

\section{A. Result Analysis with the Conventional Tracking Method}

Fig. 10 presents the variation in radiation and DC voltage based on the control method of the tracking device. Fig. 11 presents a generation comparison based on the control method of the tracking device and radiation. The program method $(40.42 \mathrm{kWh})$ is higher than the sensor method $(38.85 \mathrm{kWh})$. This is because the tracking system of the sensor method is not operating due to low radiation.

Fig. 12 presents the variation in radiation and DC voltage based on the control method of the tracking device and radiation. The average radiation is $100[\mathrm{~W} / \mathrm{m} 2]$ due to pouring rain by a typhoon on the day of experiment. Fig. 13 presents a generation comparison based on the control method of the tracking device and radiation. When the sensor method and the program method are compared, the generation of the program method $(14.17 \mathrm{kWh})$ is higher than the sensor method $(13.35 \mathrm{kWh})$. This is because the tracking system of the sensor method is not operating due to low radiation. Accordingly, in low radiation, the tracking of the program method is better than the sensor method. The analyzed data is also used to compare the proposed method with the conventional program method in section 5.2.

\section{B. Result Analysis with the Proposed Method}

Fig. 14 presents the variation in surface temperature and AC power with the proposed control method. Fig. 15 presents the variation in DC voltage and DC current with the proposed control method and the conventional control method. The DC voltage of the proposed method is higher than the conventional method. Fig. 16 presents a generation comparison of the 


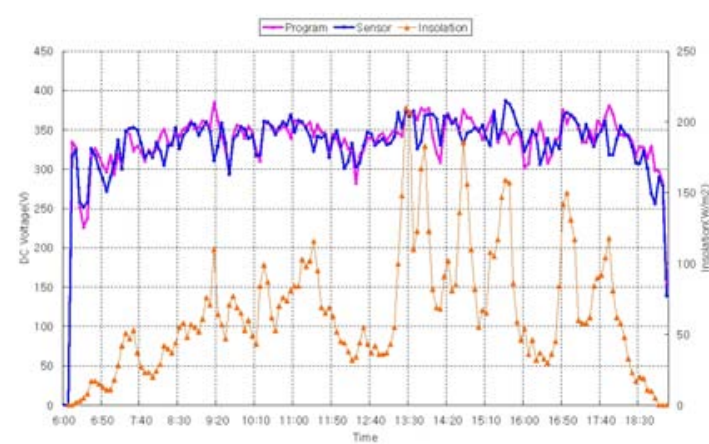

Fig. 12. Change of radiation and DC voltage by climate environment (with raining).

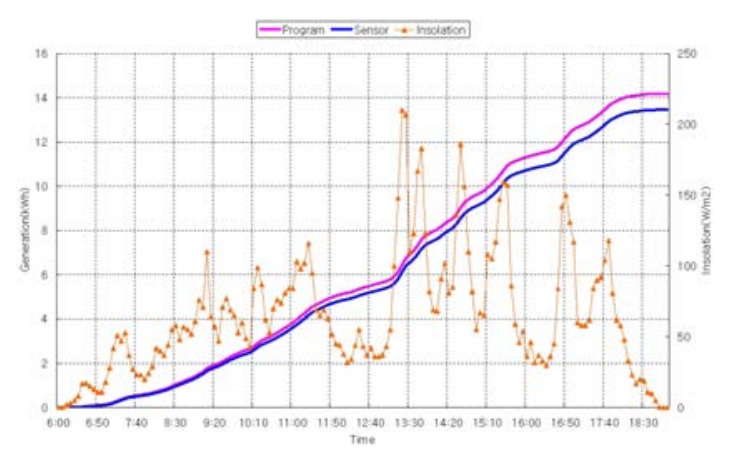

Fig. 13. Comparison with power by control method (with raining).

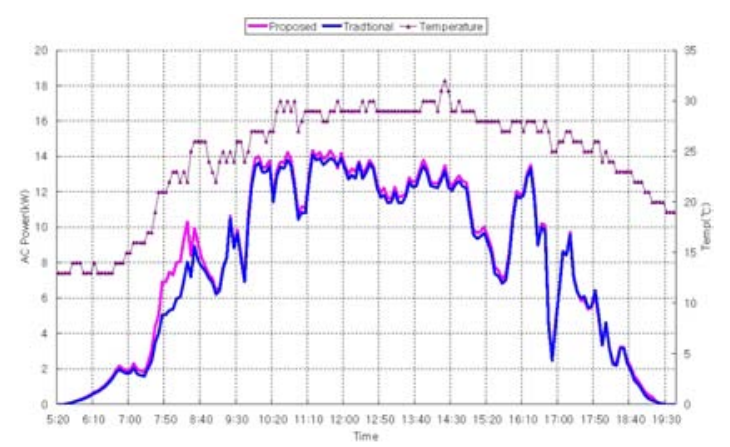

Fig. 14. Change of surface temperature and AC Power by climate environment (with proposed method).

proposed control method and the conventional control method. The generation of the non-tracking system where the tracking device is controlled to a specific location is higher than the real time tracking system in low radiation. Generation with the program method is $73.24[\mathrm{kWh}]$ and with the proposed algorithm method it is $75.11[\mathrm{kWh}]$. The generation efficiency of the proposed algorithm method is $2.5 \%$ higher than the conventional method. Accordingly, the tracking system of the proposed algorithm is better than that of the conventional method with low radiation and variations in radiation.

\section{CONCLUSIONS}

This paper proposes a novel control algorithm for a PV tracking system which can reduce unnecessary power loss in the tracking device. Sensor and program tracking PVs cannot deal with external climate factors, which causes power loss due to unnecessary operation. In particular, the sensor method

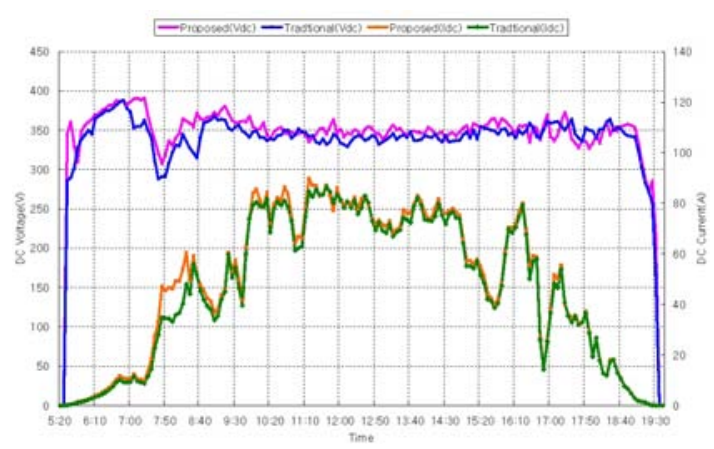

Fig. 15. Comparison with DC voltage and current by climate environment (with proposed method).

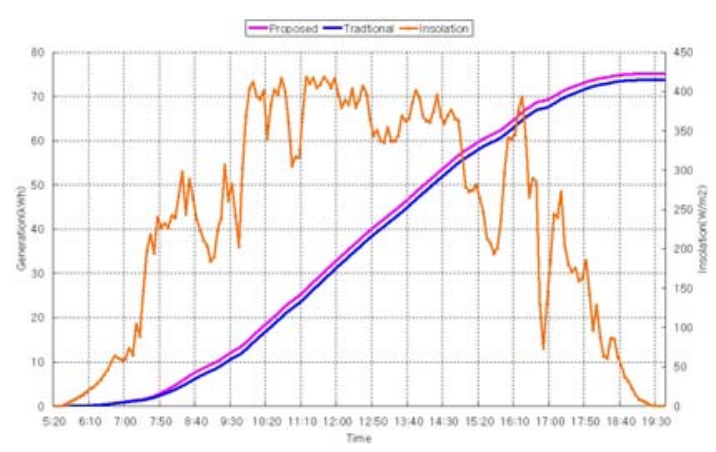

Fig. 16. Comparison with power by control method (with proposed method).

clearly reduced the generated energy by forcing a move to a preset position or by stopping at its current position under low radiation or due to a malfunction in the tracking device due to suddenly changing weather conditions such as clouds or fog.

This paper proposes an algorithm that uses radiation sensors, wind speed sensors and a rainfall cumulating sensor to quickly adapt to changes in climate. A tracking system using the proposed algorithm is compared with the conventional system in regards to DC voltage, AC voltage and power.

A tracking system with the proposed algorithm increases efficiency by about $2.5 \%$ under sudden changes or low radiation when compared to the conventional program method. Therefore, the validity of proposed algorithm has been proved.

\section{REFERENCES}

[1] R. akkaya and A. A Kulaksiz, "A microcontroller-based stand-alone photovoltaic power system for residential appliances," Elsevier, applied Energy 74, pp. 159-167, 2003.

[2] S. M. Ale-emran, M. Abedi, G. B. Gharehpetian, R. Noroozian, "Dynamic Operation of a Photovoltaic System Connected to Distribution System," International Symposium on Power Electronics, Electrical Drives, Automation and Motion, pp. 206-210, 2008.

[3] Hasan A. Yousef, "Design and Implementation of a Fuzzy Logic Computer-Controlled Sun Tracking System," Industrial Electronics, 1999. ISIE '99. Proceedings of the IEEE International Symposium, Vol.3, pp 1030-1034, 1999.

[4] Daniel A. Pritchard, "Sun Tracking by Peak Power Positioning for Photovoltaic Concentrator Arrays," Control Systems Magazine, IEEE, Vol.3, pp. 2-8, 1983.

[5] P.Roth, A. Georgiev, H. Boudinov, "Design and construction of a system for sun-tracking," Renewable Energy, Vol.29, pp. 393-402, 2004.

[6] P.Roth, A. Georgiev, H. Boudinov, "Cheap two axis sun follow device," Energy Conversion and Management, Vol.46, pp. 1179-119, 2005.

[7] W. A. Lynch, M. Salameh, "Simple eletro-optically controlled dual axis sun tracker," Solar Energy, Vol.45, pp. 65-69, 1995. 
[8] E. A. barber, H. A. Ingley, C. A. Morrison, "A solar powered tracking device for driving concentrating collectors," Alternative Energy Source, Vol.1, pp. 527-539, 1997.

[9] B. P. Edwards, "Computer based sun following system," Solar Energy, Vol.21, PP. 491-496, 1998.

[10] P. L. Swart, J. D. Van Wyk, "Source tracking and power flow control of terrestrial photovoltaic panels for concentrated sunlight," 12th IEEE Photovoltaic Specialist Conference, Vol. 1, pp. 700-795, 1998.

[11] P. O. Carden, "Steering A Field of Mirrors Using a Shared Computerbased Controller," Solar Energy, Vol.20, pp. 351-356, 1998.

[12] BLANCO-MURIEL. MANUEL, et. al., "COMPUTING THE SOLAR VECTOR,"Solar Energy, Vol. 70, No. 5, pp. 431-441, 2001.

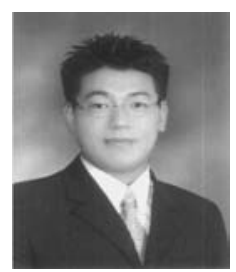

Jung-Sik Choi received his M.Eng in Electrical Control Engineering from Sunchon National University, Korea, in 2005. He is currently working toward his Ph.D. in Electrical Control Engineering at Sunchon National University, Korea. His research interests include fuzzy control, neural networks, motor control and photovoltaic power. His current research focuses on maximum power point tracking control of $\mathrm{PV}$ generation.

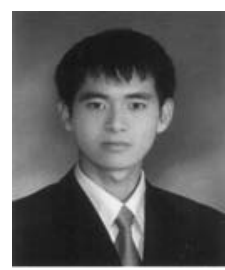

Jae-Sub Ko received his M.Eng in Electrical Control Engineering from Sunchon National University, Korea, in 2005. He is currently working toward his Ph.D. in Electrical Control Engineering at Sunchon National University, Korea. His research interests include fuzzy control, neural networks, motor control and photovoltaic power. His current research focuses on maximum power point tracking control of PV generation.

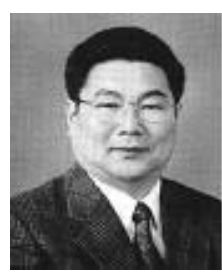

Dong-Hwa Chung received his D.Eng in Electric Engineering from Hanyang University, Korea, in 1987. $\mathrm{He}$ is a Professor of Electrical Control Engineering at Sunchon National University, Korea. His research interests include power electronics, fuzzy control, neural networks, motor control, artificial intelligent control and photovoltaic power. His current research focuses on maximum power point tracking control of PV generation. He has received about twenty awards such as the Best Paper Award and the Academy Research Award etc, from the Korean Institute of Electrical Engineers and the Korean Institute of Illuminating and Electrical Installation Engineers etc. 